\title{
August Lau
}

Finite Abelian semigroups represented into the power set of finite groups

Czechoslovak Mathematical Journal, Vol. 29 (1979), No. 1, 159-162

Persistent URL: http://dml.cz/dmlcz/101590

\section{Terms of use:}

(C) Institute of Mathematics AS CR, 1979

Institute of Mathematics of the Czech Academy of Sciences provides access to digitized documents strictly for personal use. Each copy of any part of this document must contain these Terms of use.

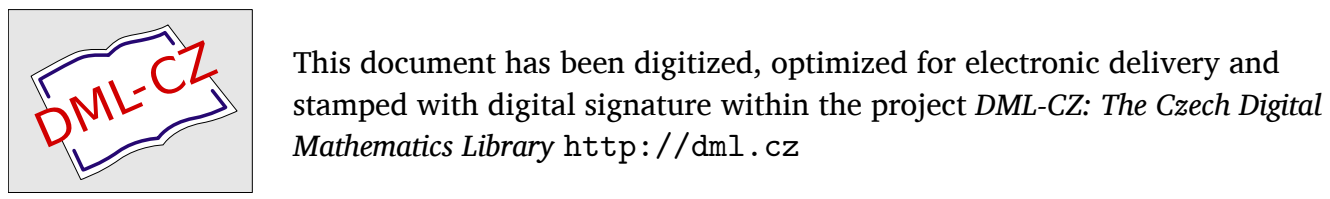




\title{
FINITE ABELIAN SEMIGROUPS REPRESENTED INTO THE POWER SET OF FINITE GROUPS
}

\author{
August Lau, Denton*)
}

(Received December 7, 1977)

Finite abelian groups have very well-defined structures and are direct sums of cyclic groups. If $2^{G}$ is the collection of nonempty subsets of a semigroup $G$, then $A B=\{a b \mid a \in A, b \in B\}$ defines a semigroup for $2^{G}$. Although finite abelian groups have been investigated, $2^{G}$ is a relatively new object for research. BYRD, LLOYD, Pederson, and StepP studied the automorphisms of $2^{G}$ (see [2]) and have made contributions to the understanding of $2^{G}$.

If one allows $G$ to be any abelian group and not just finite then TrNKová in [5] proved that every abelian semigroup is embeddable (one-to-one homomorphism) into $2^{G}$ for some abelian group $G$. But $2^{G}$ for an arbitrary abelian group is rather untractable. So further restriction was needed. In [1], BILYEU and LAU studied the collection (hyperspace) of compact subsets of a compact group and certain topological embeddings were derived.

But underlying all the general studies, a very basic question has not been settled:

Problem. If $S$ is a finite abelian semigroup, then is $S$ embeddable in $2^{G}$ for some finite abelian group $G$ ?

A finite abelian semigroup is said to be representable (in this paper) if it is embeddable in $2^{G}$ for some finite abelian group $G$. A $z$-semigroup is a semigroup having a unique idempotent which is a zero for the semigroup (see YAMADA [6] and [7]). If $S$ is a finite semigroup, then it has a minimal ideal denoted by $M(S)$ and $S / M(S)$ is the Rees quotient. If $S$ has an identity 1 , then $H(1)$ is the group of units.

We were not able to solve the general problem but were able to prove that if finite abelian $z$-semigroups are representable, then finite abelian semigroups are representable. The following lemmas are helpful to establish this fact.

*) The research was supported by North Texas State University Faculty Research Grant (1977). 
Lemma 1. If $G_{1}, \ldots, G_{n}$ are finite groups, then $\prod_{i=1}^{n} 2^{G_{i}}$ is embeddable in $2^{n G_{i}}$.

Proof. Use the function which sends $\left(A_{1}, \ldots, A_{n}\right)$ to $A_{1} \times \ldots \times A_{n}$.

Lemma 2. If $S$ is a finite abelian semigroup and for each pair $x \neq y$ in $S$, there is a homomorphism $f$ from $S$ into $2^{G}$ for some finite abelian group $G$ so that $f(x) \neq$ $\neq f(y)$, then $S$ is representable.

Proof. Since there are finitely many homomorphisms from $S$ into $2^{G_{1}}, \ldots, 2^{G_{n}}$ to separate points, then $S$ is embeddable in $\prod 2^{G_{i}}$, hence in $2^{\Pi G_{i}}$ by Lemma 1 .

Lemma 3. If $S, T$ are semigroups and $i: S \rightarrow T$ is a one-to-one homomorphism, then $i^{*}: 2^{S} \rightarrow 2^{T}$ is a one-to-one homomorphism where $i^{*}(A)=i(A)$.

Lemma 4. If $S$ is a semigroup and $\sigma: 2^{2^{S}} \rightarrow 2^{S}$ is defined by $\sigma(\mathscr{A})=\bigcup\{A \mid A \in \mathscr{A}\}$, then $\sigma$ is a homomorphism.

Theorem. If each finite abelian z-semigroup is representable, then every finite abelian semigroup is representable.

Proof. Induct on the order of $S$ where $S$ is a finite abelian semigroup. Suppose $M(S)$ has more than one element. Let $e=e^{2} \in M(S)$. Note that $M(S)$ is a group since $S$ is abelian. Then $f: S \rightarrow M(S)$ by $f(x)=x e$ and $p: S \rightarrow S / M(S)$ would separate points. But $S / M(S)$ has an order less than that of $S$. By induction, $S / M(S)$ is representable.

We can now assume that $S$ has a zero. Choose $e=e^{2} \neq 0$ so that it is minimal with respect to the idempotent ordering of all nonzero idempotents. Again $f: S \rightarrow S e$ by $f(x)=x e$ and $S \rightarrow S / S e$ separate points. Hence we can assume that $S=S e$, i.e., $S$ has an identity 1 and has only two idempotents 0 and 1 .

Suppose $H(1)=\{1\}$. Then $I=S \backslash H(1)$ is a finite abelian $z$-semigroup. Let $j$ be an embedding of $I$ into $2^{G}$ for some finite abelian group $G$. Let $H$ be a finite abelian group having more than one element. Then $J: S \rightarrow 2^{G \times H}$ defined by:

is an embedding.

$$
J(x)=\left\{\begin{array}{lll}
j(x) \times H & \text { if } & x \neq 1, \\
\{(1,1)\} & \text { if } & x=1,
\end{array}\right.
$$

Assume that the set of idempotents of $S$ is $\{0,1\}$ and $H(1) \neq\{1\}$.

Let $H=H(1)$. Since $|I \cup\{1\}|<|S|$, then by induction, we have $j: I \cup\{1\} \rightarrow 2^{G}$ an embedding for some finite abelian group $G$. Let

1. $J: H \times(I \cup\{1\}) \rightarrow H \times 2^{G}$ be defined by $J(h, x)=(h, j(x))$,

2. $K: H \times 2^{G} \rightarrow 2^{H \times G}$ be defined by $K(h, A)=\{h\} \times A$,

3. $m: H \times(I \cup\{1\}) \rightarrow S$ be defined by $m(h, x)=h x$. 
Then

$$
m^{-1}(x)=\left\{\begin{array}{l}
\left\{\left(h, h^{-1} x\right) \mid h \in H\right\} \quad \text { if } \quad x \in I, \\
\{(x, 1)\} \text { if } \quad x \in H(1) .
\end{array}\right.
$$

Claim. $M: S \rightarrow 2^{H \times(I \cup\{1\})}$ is a homomorphism where $M(x)=m^{-1}(x)$.

Let $x, y \in S$. Then $M(x) M(y) \subseteq M(x y)$ since $m$ is a homomorphism.

Case A. Suppose $x \in H$ and $y \in I$. Then $x y \in I$. Let $(h, z) \in M(x y)$. Then $h z=x y$, $m^{-1}(x)=(x, 1)$ and $(h, z)=(x, 1)\left(h x^{-1}, z\right) \in M(x) M(y)$.

Case B. Suppose $x \in H$ and $y \in H$. Then $M(x y)=(x y, 1)=(x, 1)(y, 1)=$ $=M(x)^{-M}(y)$.

Case C. Suppose $x, y \in I$. Let $(h, z) \in M(x y)$. Then $h z=x y$. Hence $(h, z)=$ $=\left(h, h^{-1} x\right)(1, y) \in M(x) M(y)$.

Consider $i: S \rightarrow 2^{H \times G}$ defined by composing these four functions:

$$
S \rightarrow^{M} 2^{H \times(I \cup\{1\})} \rightarrow^{J^{*}} 2^{H \times 2^{G}} \rightarrow^{K^{*}} 2^{2^{H \times G}} \rightarrow^{\sigma} 2^{H \times G} .
$$

We shall prove that $i=\sigma K^{*} J^{*} M$ is an embedding. It is clear that it is a homomorphism.

Case 1. Let $x, y \in I$.

$$
\begin{gathered}
i(x)=\sigma K^{*} J^{*} M(x)=\sigma K^{*} J^{*}\left\{\left(h, h^{-1} x\right) \mid h \in H\right\}=\sigma K^{*}\left\{\left(h, j\left(h^{-1} x\right)\right) \mid h \in H\right\}= \\
=\sigma\left\{\{h\} \times j\left(h^{-1} x\right) \mid h \in H\right\}=\bigcup_{h \in H}\{h\} \times j\left(h^{-1} x\right) . \\
i(y)=\bigcup_{h \in H}\{h\} \times j\left(h^{-1} y\right) .
\end{gathered}
$$

Suppose $i(x)=i(y)$. Then $\{1\} \times j(x) \subseteq \bigcup_{h \in H}\{h\} \times j\left(h^{-1} y\right)$. Hence $\{1\} \times j(x) \subseteq$ $\subseteq\{1\} \times j(y)$. Conversely, $\{1\} \times j(y) \subseteq\{1\} \times j(x)$. But $j(x)=j(y)$ implies $x=y$.

Case 2. Let $x, y \in H$.

$$
\begin{gathered}
i(x)=\sigma K^{*} J^{*} M(x)=\sigma K^{*} J^{*}\{(x, 1)\}=\sigma K^{*}\{(x, j(1))\}= \\
=\sigma\{\{x\} \times j(1)\}=\{x\} \times j(1) . \\
i(y)=\{y\} \times j(1) .
\end{gathered}
$$

Hence $i(x)=i(y)$ implies $x=y$.

Case 3. Let $x \in H, y \in I$. Then

and

$$
i(x)=\{x\} \times j(1)
$$

$$
i(y)=\bigcup_{h \in \boldsymbol{H}}\{h\} \times j\left(h^{-1} y\right) .
$$


Hence $i(x) \neq i(y)$ since $H$ has more than one element.

Remark. Left zero semigroups $(x y=x$ for all $x, y)$ are not embeddable in $2^{G}$ for any finite group $G$. Hence the commutative property of the semigroup is important to the problem.

Remark. The structure of finite abelian $z$-semigroups was thoroughly discussed in [6] and [7] but we are still unable to solve the general problem.

\section{References}

[1] R. G. Bilyeu and A. Lau: Representations into the hyperspace of a compact group, Semigroup Forum, Vol. 13 (1977), 267-270.

[2] R. D. Byrd, J. T. Lloyd, F. D. Pedersen, J. W. Stepp: Automorphisms of semigroups of complexes of Abelian groups, Bull. Amer. Math. Soc., Vol. 83 \# 2 (1977), 260-261.

[3] H. B. Mann: Addition theorems: the addition theorems of group theory and number theory, Interscience Pub. (1965).

[4] L. Redei: The theory of finitely generated commutative semigroups, Pergamon Press (1965).

[5] V. Trnková: On a representation of commutative semigroups, Semigroup Forum, Vol. 10 \# 3 (1975), 203-214.

[6] M. Yamada: Construction of finite commutative $z$-semigroups, Proc. Japan Acad. Vol. 40 \# 2 (1964), 94-98.

[7] M. Yamada: Construction of finite commutative semigroups, Bull. of Shímane Univ., No. 15 (1965), 1-11.

Author's address: Department of Mathematics, North Texas State University, Denton, Texas 76203, U.S.A. 